\title{
EXACT, APPROXIMATE SOLUTIONS AND ERROR BOUNDS FOR COUPLED IMPLICIT SYSTEMS OF PARTIAL DIFFERENTIAL EQUATIONS
}

\author{
LUCAS JÓDAR \\ Departamento de Matemática Aplicada \\ Universidad Politécnica \\ P.0. Box 22.012 Valencia 46071 \\ Spain \\ (Received July 2, 1991 and in revised form October 17, 1991)
}

\begin{abstract}
In this paper coupled implicit initial-boundary value systems of second order partial differential equations are considered. Given a finite domain and an admissible error $\varepsilon$ an analytic approximate solution whose error is upper bounded by $\varepsilon$ in the given domain is constructed in terms of the data.
\end{abstract}

KEY WORDS AND PHRASES. Implicit coupled partial differential system, approximate solution, error bound, Padé approximant.

1991 AMS SUBJECT CLASSIFICATION CODES. 35A20, 35A40, 65N15, 65P05

\section{INTRODUCTION}

Many physical systems can not be described by a single equation but, in fact are modeled by a coupled system of equations. Coupled systems of second order partial differential equations occur in various fields of applied sciences such as fluid mechanics, theory of elasticity, wave propagation, nonequilibrium thermodynamics, etc. Examples of such type of different coupled systems of partial, differential equations may be found in $[2,3,6,8,10,13,18]$. Methods to study such coupled systems based on the transformation of the original system into a system of independent or uncoupled partial differential equations may be found in $[4,5,16]$, for instance. However the well-adapted technique of such transformations, called the method of elimination has some basic drawbacks as it has been shown in [5]. This motivates the search of some alternative which avoid the inconveniences and straightforward calculations involved in the method of elimination. In a recent paper [9] a method is proposed to solve coupled systems of partial differential equations avoiding uncoupling techniques. The aim of this paper is to construct analytic approximate solutions for a more general type of equations than the one considered in |9|.

This paper is organized as follows. In section 2 we provide a series solution of coupled systems of the type

$$
\begin{aligned}
& \mathrm{AU}_{x x}(x, t)-U_{t}(x, t)=b, \quad 0<x<a, \quad t>0 \\
& U(0, t)=t b_{1}, U(a, t)=t b_{2}, \quad t>0
\end{aligned}
$$




$$
U(x, 0)=F(x), \quad 0 \leqq x \leqq a
$$

where $U=\left(u_{1}, \ldots, u_{m}\right)^{T}, b, b_{1}, b_{2}$ and $F(x)$ are vectors in $\mathbb{R}^{m}$. A is a matrix in $\mathbb{R}^{m \times m}$ such that

$$
\mathrm{b}, \mathrm{b}_{1} \text { and } \mathrm{b}_{2} \text { belong to the range of } \mathrm{A}
$$

The real part of each eigenvalue $z$ of $A$ satisfies $\operatorname{Re}(z) \geqq 0$

By means of a matrix separation of variables method, a convergent series solution of the problem is given. Section 3 is concerned with the construction of a finite and computable approximate solution of the problem (1.1)-(1.5), such that its approximation error be smaller than a prefixed admissible error $\varepsilon$. This is perfomed by truncation of the infinite series and the approximation of certain matrix exponentials by Padé approximants of an appropriate degree.

Coupled systems of the type (1.1) with A singular appear in the study of the Hodgkin-Maxley nervous conduction equation $[14\rceil,[15\rceil$. For the sake of clarity in the presentation we recal1 some concepts and well known results about matrix norms. If $C$ is a matrix in $\mathbb{R}^{\mathrm{mxm}}$, we denote by $\|C\|$ its operator norm defined as the quare root of the maximum eigenvalue of $C^{T} C$ where $C^{T}$ is the transpose matrix of $C,\{12\}, p .14$. The $\infty$-norm of $C$, denoted by $\|C\|_{\infty}$ is defined by

$$
\|C\|_{\infty}=\max _{i}\left\{\sum_{j=l}^{m}\left|c_{i j}\right|\right\}
$$

and we recall that from $\mid 7], p .15$, it follows that

$$
\mathrm{m}^{-\frac{1}{2}}\|\mathrm{C}\|_{\infty} \leqq\|C\| \leqq \mathrm{m}^{\frac{1}{2}}\|\mathrm{C}\|_{\infty}
$$

The set of all eigenvalues of $\mathrm{C}$ is denoted by $\sigma(C)$ and the identity matrix in $\mathbb{R}^{\mathrm{mxm}}$ is denoted by $I$.

2. A SERIES SOLUTION OF THE PROBLEM.

Taking into account the hypothesis $(1.4)$, let us take vectors $c_{, c_{1}}$ and $c_{2}$ in $\mathbb{R}^{\mathrm{m}}$ such that

$$
\mathrm{b}=\mathrm{Ac}, \quad \mathrm{b}_{1}=\mathrm{Ac} \mathrm{c}_{1} \quad \text { and } \quad \mathrm{b}_{2}=\mathrm{Ac}_{2}
$$

An easy computation shows that

$$
Z(x)=\frac{1}{2} x(x-a) c
$$

is a solution of the problem

$$
\mathrm{AZ}^{\prime \prime}(\mathrm{x})=\mathrm{b}, \quad \mathrm{Z}(0)=\mathrm{Z}(\mathrm{a})=0
$$

On the other hand let $V(x, t)$ be defined by

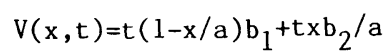

and note that $V$ satisfies the problem

$$
\begin{aligned}
& A V_{x x}(x, t)-V_{t}(x, t)=\left(b_{1}-b_{2}\right) x / a-b_{1}, \quad 0<x<a, \quad t>0 \\
& V(0, t)=t b_{1}, \quad V(a, t)=t b_{2}, \quad t>0 \\
& V(x, 0)=0, \quad 0 \leqq x \leqq a
\end{aligned}
$$


Now let us consider the function

$$
T(x)=(1 / 6)\left\{\left(c_{2}-c_{1}\right) x^{3} / a+3 c_{1} x^{2}-\left(c_{2}+2 c_{1}\right) a x\right\}
$$

that satisfies

$$
\mathrm{T}^{\prime \prime}(\mathrm{x})=\mathrm{c}_{1}+\left(\mathrm{c}_{2}-\mathrm{c}_{1}\right) \mathrm{x} / \mathrm{a} ; \mathrm{T}(0)=\mathrm{T}(\mathrm{a})=0
$$

and from (2.1),

$$
\operatorname{AT}^{\prime \prime}(\mathrm{x})=\mathrm{b}_{1}+\left(\mathrm{b}_{2}-\mathrm{b}_{1}\right) \mathrm{x} / \mathrm{a}, \quad \mathrm{T}(0)=\mathrm{T}(\mathrm{a})=0
$$

From the previous comments, if $W(x, t)$ satisfies the problem

$$
\begin{aligned}
& A W_{x x}(x, t)-W_{t}(x, t)=0, \quad 0<x<a, \quad t>0 \\
& W(0, t)=0, \quad W(a, t)=0, \quad t>0 \\
& W(x, 0)=F(x)-Z(x)+T(x), \quad 0 \leq x \leq a
\end{aligned}
$$

then $U(x, t)$ defined by

$$
U(x, t)=W(x, t)+V(x, t)-T(x)+Z(x)
$$

is a solution of the problem (1.1)-(1.3).

If we look for solutions $W(x, t)$ of the homogeneous boundary value problem (2.10) $(2.11)$, of the form

$$
W(x, t)=S(t) X(x), \quad S(t) \varepsilon \mathbb{R}^{m x m}, X(x) \varepsilon \mathbb{R}^{m}
$$

and from section 3 of $[9]$, one obtains a family of solutions $W_{n}(x, t)$ of the problem $(2.10)-(2.11)$, of the form

$$
W_{n}(x, t)=\exp \left(-(n \pi / a)^{2} A t\right) \sin (n \pi x I / a) d_{n}, \quad d_{n} \in \mathbb{R}^{m}
$$

If we denote by $G(x)$ the vector function

$$
G(x)=F(x)-Z(x)+T(x)
$$

and we superpose the functions $w_{n}(x, t)$ given by $(2.15)$, then a formal solution of the problem $(2.10)-(2.12)$ is given by

$$
W(x, t)=\sum_{n \geq 1} W_{n}(x, t)
$$

where

$$
d_{n}=(2 / a) \int_{0}^{a} G(x) \sin (n \pi x / a) d x
$$

In order to prove that $W(x, t)$ defined by $(2.15),(2.17),(2.18)$, is a solution of the problem (2.10),(2.12), we are going to show that under which conditions such series $\mathrm{W}(\mathrm{x}, \mathrm{t})$ defines a continuous vector function admits termwise partial derivatives, once with respect to the variable $t$ and twice with respect to the variable $x$. Note that for the case where the real part of each eigenvalue $z$ of the matrix $A$ has a positive real part, in [9] we proved that in fact $W(x, t)$ is a solution of $(2.10),(2.12)$. 
In this paper we assume that the matrix $A$ is singular and satisfies $(1.5)$, so let us denote the spectrum $\sigma(A)=\left\{\lambda_{1}, \ldots, \lambda_{r}, \lambda_{r+1}, \ldots, \lambda_{s}\right\}$, such that $\lambda_{i} \neq \lambda_{j}$ for $i \neq j$ and

$$
0=\operatorname{Re}\left(\lambda_{1}\right)=\operatorname{Re}\left(\lambda_{2}\right)=\ldots=\operatorname{Re}\left(\lambda_{r}\right)<\operatorname{Re}\left(\lambda_{r+1}\right) \leq \operatorname{Re}\left(\lambda_{r+2}\right) \leq . . \leq \operatorname{Re}\left(\lambda_{s}\right)
$$

and let us denote by $m_{i}$ the index of the eigenvalue $\lambda_{i},[19], p .556$. If $E\left(\lambda_{k}\right)$ denotes the spectral projection associated to the eigenvalue $k$ of the matrix $A$ and if for a fixed $t>0$, we consider the complex function $f_{n}(z)=\exp \left(-t z(n \pi / a)^{2}\right)$ then from theorem 8 of $[19], p .559$, it follows that

$$
\begin{gathered}
\exp \left(-t A(n \pi / a)^{2}\right)=\sum_{k=1}^{s} \sum_{j=0}^{m_{k}-1}\left(A-\lambda_{k} I\right)^{j} E\left(\lambda_{k}\right) f_{k j}(n), \\
f_{k j}(n)=f_{n}^{(j)}\left(\lambda_{k}\right) / j !=(n \pi / a)^{2 j}(-1)^{j} \exp \left(-t \lambda_{k}(n \pi / a)^{2}\right) t^{j} / j !
\end{gathered}
$$

Hence the series $W(x, t)$ defined by $(2.15),(2.17),(2.18)$, will be convergent and admit termwise partial differentiation if each of the series

$$
Z_{k, j}(x, t)=\sum_{n \geq 1}\left(A-\lambda_{k} I\right)^{j_{E}}\left(\lambda_{k}\right) \sin (n \pi x I / a) f_{k j}(n) d_{n}
$$

verifies the same properties for $1 \leq k \leq s, 0 \leq j \leq m_{k}-1$.

Let us denote by $I\left(t_{0}, t_{1}\right)$ the rectangle $[0, a] x\left[t_{0}, t_{1}\right]$ and 1 et us introduce the constants

$$
\begin{gathered}
C=\max \left\{C_{k j} ; 1 \leq k \leq s, 0 \leq j \leq m_{k}-1\right\} ; C_{k j} \geq\left\|\left(A-\lambda_{k} I\right)^{j} E\left(\lambda_{k}\right) / j !\right\| \\
J=\max \left\{2\left(m_{k}-1\right) ; 1 \leq k \leq r\right\}
\end{gathered}
$$

Let $\rho>0$ such that $\operatorname{Re}\left(\lambda_{i}\right) \geq \rho>0$, for $r+1 \leq i \leq s$, then from $(2.21)$, if $(x, t) \varepsilon I\left(t_{0}, t_{1}\right)$ it follows that

$$
\begin{gathered}
\left|f_{k j}(n)\right| \leqq(n \pi / a)^{2 j} \exp \left(-t_{o} \operatorname{Re}\left(\lambda_{1}\right)(n \pi / a)^{2} C_{k j}\left(t_{1}\right)^{j}, r+1 \leq k \leqq s\right. \\
\left|f_{k j}(n)\right| \leqq(n \pi / a)^{2 j} C_{k j}\left(t_{1}\right)^{j}, \quad 1 \leq k \leq r
\end{gathered}
$$

If we denote by $G_{n}(x, t)$ the general term of the series $Z_{k, j}(x, t)$ defined by $(2.22)$ for $(x, t) \varepsilon I\left(t_{0}, t_{1}\right)$, it follows that

$$
\begin{gathered}
\left\|G_{n}(x, t)\right\| \leq C_{k j}\left(t_{1} \pi^{2} / a^{2}\right)^{j}{ }^{2 j}\left\|d_{n}\right\|, \quad 1 \leq k \leq r \\
\left\|G_{n}(x, t)\right\| \leq C_{k j}\left(t_{1} \pi^{2} / a^{2}\right)^{j} \exp \left(-\rho t_{o}(n \pi / a)^{2}\right) n^{2 j}\left\|d_{n}\right\|, \quad r+1 \leq k \leq s
\end{gathered}
$$

In both cases we have

$$
\left\|G_{n}(x, t)\right\| \leq C_{k j}\left(t \pi_{1}^{2} / a^{2}\right)^{J}\left\|d_{n}\right\| n^{J}, \quad 1 \leq k \leq s
$$

where $\mathrm{J}$ is defined by $(2.24)$. 
Now let us assume that the function $G(x)$ defined by (2.16) is $J+4$ times continuously differentiable in $|0, a|$ such that

$$
G^{(i)}(0)=G^{(i)}(a)=0, \quad 0 \leqq i \leqq J+4
$$

then from $|17|, p .71$, the odd 2a-periodic extension of $G(x)$ in the real line admits $\mathrm{J}+4$ continuous derivatives and there exists a constant $M$ defined by

$$
M=\sup \left\{\left\|G^{(J+4)}(x)\right\| ; 0 \leqq x \leqq a\right\}
$$

such that

$$
\left\|d_{n}\right\| \leqq n^{-(J+4)}, n \geqq 1
$$

On the other hand the general term of the series obtained by termwise partial differentiation with respect to $x$ and twice with respect to $x$, are upper bounded by a sequence of the type $0\left(n^{J+2}\right)\left\|d_{n}\right\|$, as $n \rightarrow \rightarrow \infty$, uniformly for $(x, t) \varepsilon I\left(t_{0}, t_{1}\right)$. Thus from the Weierstrass mayorant criterion and the derivation theorem for functional series $\mid 1$, theorem $9.14 \mid$, the series $W(x, t)$ defined by $(2.15),(2.17),(2.18)$, defines a continuous function which admits second order partial derivatives with respect to $x$ and first order partial derivatives with respect to the variable $t$, calculated by termwise partial differentiation in (2.17). From the previous comments the following result has been established:

THEOREM 1. Let us consider the initial-boundary value problem $(1.1)-(1.5)$, let $Z(x), T(x)$ and $V(x, t)$ be defined by $(2.2),(2.8)$ and $(2.4)$, respectively. If the eigenvalues of $A$ are ordered by (2.19) and $J$ is defined by (2.24), assuming that $G(x)$ defined by (2.16) satisfies the condition (2.30), then a solution of the problem (1.1)$(1.5)$, is given by $(2.13)$, where $W(x, t)$ is defined by $(2.15),(2.17)$ and $(2.18)$.

REMARK 1. If $A$ is a nonsingular matrix and satisfies $\operatorname{Re}(z)>0$ for each eigenvalue of $A$, then the condition (1.4) is obviously satisfied. Furthermore in this case it is unnecesary to impose the differentiability condition (2.30) because of the inequality (2.25). Thus the results of sections 2 and 3 of $|9|$ are a consequence of the previous comments. Concerning to the differentiability of $G(x)$ and the condition (2.30), it is important to point out that if we do not impose the condition (2.30), from $[17], p .71$, we only can assure that the Fourier sine coefficients $d_{n}$ defined by (2.18) satisfy an inequality of the type

$$
\left\|d_{n}\right\| \leq M_{1} / n+M_{2} / n^{2}+\ldots+M_{J+4} / n^{J+4}
$$

Note that if $\mathrm{A}$ is a non diagonalizable matrix then the problem (1.1)-(1.3) can not be uncoupled and the uncoupling techniques are not useful to solve the problem.

3. APPROXIMATE SOLUTIONS AND ERROR BOUNDS

The solution $U(x, t)$ provided by theorem 1 has some numerical drawbacks, first of all $U(x, t)$ is an infinite series and secondly, its general term involves the computation of matrix exponentials what is not an easy task, 111$\}$. In this section we try to avoid these inconveniences by truncating the infinite series and after approximating in the finite series the matrix exponentials by Padé approximants of an appropriate degree. 
Let $q$ be a positive integer $q>1$ and $\operatorname{let} \mathrm{N}_{\mathrm{qq}}(\mathrm{z})$ be the polynomial

$$
N_{q q}(z)=\sum_{k=0}^{q} \frac{(2 q-k) ! q ! z^{k}}{(2 q) ! k !(q-k) !}
$$

and let us consider the Padé function $\mathrm{R}_{\mathrm{qq}}(\mathrm{z})=\left(\mathrm{N}_{\mathrm{qq}}(-\mathrm{z})\right)^{-1} \mathrm{~N}_{\mathrm{qq}}(\mathrm{z})$. If $\mathrm{C}$ is a matrix in $\mathbb{R}^{\mathrm{mxm}}$ and $\mathrm{j}$ is an integer such that

$$
2^{j-1} \geqq\|C\|_{\infty}
$$

and we define

$$
\begin{aligned}
& F_{q q}(C)=\left|R_{q q}\left(C / 2^{j}\right)\right|^{2^{j}}, \quad q>1 \\
& \gamma=\frac{2^{3-2 q}(q !)^{2}}{(2 q) !(2 q+1) !}
\end{aligned}
$$

From $\mid 7$, p.398| it follows that

$$
\left\|\exp (C)-F_{q q}(C)\right\|_{\infty} \leq \gamma\|C\|_{\infty} \exp \left(2\|C\|_{\infty}\right)
$$

Let $t_{0}, t_{1}$ and $\varepsilon$ be positive numbers and let us consider the domain $I\left(t_{0}, t_{1}\right)$. Since $m_{1}+m_{2}+\ldots+m_{s} \leqq m$, from $(2.20)$ and (2.29) it follows that

$$
\left\|\exp \left(-t A(n \pi / a)^{2}\right)\right\| \leq m(\pi / a){ }^{J} J^{J} C\left(t_{1}\right)^{m}
$$

Now let $n_{0}$ be the first positive integer satisfying

$$
\sum_{n>n_{0}} n^{-4} \leq(\varepsilon / 2)\left\{C M\left(t_{1}\right)^{m}(\pi / a)^{J}\right\}^{-1}
$$

Taking into account that $\|\sin (n \pi x I / a)\| \leq 1$ and from (2.32), if we denote by $w\left(x, t, n_{0}\right)$ the $n_{0}$-th partial sum of the series (2.17), then it follows that

$$
\left\|W(x, t)-W\left(x, t, n_{0}\right)\right\| \leq \varepsilon / 2, \quad(x, t) \varepsilon I\left(t_{0}, t_{1}\right)
$$

Let $j$ be a positive integer $j>1$ such that.

$$
2^{j-1} \geq\left(n_{0} \pi / a\right)^{2} t_{1}\|A\|_{\infty}
$$

If for $t \varepsilon\left[t_{0}, t_{1}\right]$ we define

$$
F_{q q}(n, t)=F_{q q}\left(-(n \pi / a)^{2} t A\right)=\left\lfloor R_{q q}\left(-(n \pi / a)^{2} t A / 2^{j}\right\rfloor^{2^{j}}\right.
$$

from (3.1)-(3.3) it follows that

$\left\|\exp \left(-(n \pi / a)^{2} t A\right)-F_{q q}(n, t)\right\|_{\infty} \leq t y(n \pi / a)^{2}\|A\|_{\infty} \exp \left(2(n \pi / a)^{2} t\|A\|_{\infty}\right)$

Hence from (2.32), if $(x, t) \varepsilon I\left(t_{0}, t_{1}\right)$ it follows that 


$$
\begin{aligned}
& \sum_{n=1}^{n_{0}}\left\|F_{q q}(n, t)-\exp \left(-t A(n \pi / a)^{2}\right)\right\|_{\infty}\left\|\sin (n \pi x I / a) d_{n}\right\|_{\infty} \\
& \leq M \gamma t_{1}\|A\|_{\infty} \sum_{n=1}^{n_{0}} n^{-(J+2)}
\end{aligned}
$$

Now let us take $q$ enough large so that $\gamma$ defined by (3.2) satisfies

$$
\gamma<\frac{\left(M t_{1}\|A\|_{\infty} m^{\frac{1}{2}}\right)^{-1} \varepsilon}{2 \sum_{n=1}^{n_{0}} n^{-(J+2)} \exp \left(2(n \pi / a)^{2} t_{1}\|A\|_{\infty}\right)}
$$

and let us denote by $F\left(x, t, n_{0}, q\right)$ the vector function defined by

$$
F\left(x, t, n_{0}, q\right)=\sum_{n=1}^{n_{0}} F_{q q}(n, t) \sin (n \pi x I / a) d_{n}
$$

From $(1.6),(3.10)$ and $(3.11)$, we have

$$
\left\|W\left(x, t, n_{0}\right)-F\left(x, t, n_{0}, q\right)\right\| \leq \varepsilon / 2, \quad(x, t) \varepsilon I\left(t_{0}, t_{1}\right)
$$

Now from (3.6) and (3.13), it follows that

$$
\left\|W(x, t)-F\left(x, t, n_{0}, q\right)\right\| \leq \varepsilon
$$

and from theorem 1 and the previous comments the following result has been established

THEOREM 2. Let us consider the hypotheses and the notation of theorem 1 , let $\varepsilon$ and $t_{0}$ be positive numbers and let $I\left(t_{0}, t_{1}\right)=[0, a] x\left|t_{0}, t_{1}\right|$. If $j$ is defined by (3.7) $n_{0}$ is defined by (3.5) and if $q$ is chosen so that satisfies (3.11) and $F\left(x, t, n_{0}, q\right)$ is the finite series

$$
F\left(x, t, n_{0}, q\right)=\sum_{n=1}^{n_{0}} F_{q q}(n, t) \sin (n \pi x I / a) d_{n}
$$

where $F_{q q}(n, t)$ is defined by $(3.8)$, and $U(x, t)$ is the exact solution of the problem $(1.1)-(1.5)$, provided by theorem 1 . Then the function

$$
U\left(x, t, n_{0}, q\right)=F\left(x, t, n_{0}, q\right)+V(x, t)-T(x)+Z(x)
$$

where $V(x, t), Z(x)$ and $T(x)$ are defined by $(2.4),(2.2)$ and (2.8) respectively, is an approximate solution of the problem (1.1)-(1.5), such that

$$
\left\|U\left(x, t, n_{0}, q\right)-U(x, t)\right\| \leq \varepsilon \text {, uniformly for }(x, t) \varepsilon I\left(t_{0}, t_{1}\right)
$$

In the following we give an example of the problem (1.1)-(1.5), where the matrix $A$ is singular and non diagonalizable, thus the uncoupling technique proposed in [16] is not available. 
EXAMPLE. Let us consider the problem $(1.1)-(1.3)$, where $m=3, a=\pi, t_{0}=0, t_{1}=1 / 4$

$$
\begin{gathered}
A=\left|\begin{array}{ccc}
\frac{1}{2} & \frac{1}{2} & 0 \\
-\frac{1}{2} & 3 / 2 & 0 \\
0 & 0 & 0
\end{array}\right|, \quad b=\left|\begin{array}{l}
1 \\
1 \\
0
\end{array}\right|, \quad b_{1}=\left|\begin{array}{c}
\frac{1}{2} \\
-\frac{1}{2} \\
0
\end{array}\right|, \quad b_{2}=\left|\begin{array}{c}
\frac{1}{2} \\
3 / 2 \\
0
\end{array}\right| \\
F(x)=\left|\begin{array}{c}
\frac{(x(x-\pi))^{7}}{2 \pi^{8} 8 !}+\frac{x^{3}}{6 \pi}-\frac{x^{2}}{2}-\frac{\pi x}{3} \\
\frac{(x(x-\pi))^{7}}{4 \pi^{8} 8 !}-\frac{x^{3}}{6 \pi}+\frac{\pi x}{6} \\
\frac{(x(x-\pi))^{7}}{2 \pi^{8} 8 !}
\end{array}\right|
\end{gathered}
$$

It is easy to show that vectors $b, b_{1}$ and $b_{2}$ belong to the range of the matrix $A$ and that $A c=b, A c_{1}=b_{1}, A c_{2}=b_{2}$, where

$$
c=\left|\begin{array}{l}
1 \\
1 \\
0
\end{array}\right|, \quad c_{2}=\left|\begin{array}{l}
0 \\
1 \\
0
\end{array}\right|, \quad c_{1}=\left|\begin{array}{l}
1 \\
0 \\
0
\end{array}\right|
$$

The spectrum of the matrix $A$ is $\sigma(A)=\{0,1\}$, and the index of $\lambda_{1}=0$ is $m_{1}=1, \lambda_{2}=1$, $m_{2}=2$. The spectral projections associated to the matrix $A$ are

$$
E\left(\lambda_{1}\right)=\left[\begin{array}{lll}
0 & 0 & 0 \\
0 & 0 & 0 \\
0 & 0 & 1
\end{array}\right], \quad E\left(\lambda_{2}\right)=\operatorname{I}-E\left(\lambda_{1}\right)=\left|\begin{array}{lll}
1 & 0 & 0 \\
0 & 1 & 0 \\
0 & 0 & 0
\end{array}\right|
$$

It is easy to show that the constant $C$ given by (2.23) is $C=1$ and that $\|A\|_{\infty}=2$. The solution $\mathrm{Z}(\mathrm{x})$ of the corresponding problem $(2.3)$ is given by

$$
Z(x)=\frac{1}{2}(x-\pi) x\left[\begin{array}{l}
1 \\
1 \\
0
\end{array}\right]
$$

The functions $V(x, t)$ and $T(x)$, defined by (2.4) and (2.8) respectively, take the form

$$
\begin{aligned}
& \left.V(x, t)=t(1-x / \pi) \mid \begin{array}{c}
\frac{1}{2} \\
-\frac{1}{2} \\
0
\end{array}\right]+\frac{t x}{\pi}\left|\begin{array}{c}
\frac{1}{2} \\
3 / 2 \\
0
\end{array}\right| \\
& T(x)=(1 / 6)\left\{\left|\begin{array}{c}
-1 \\
1 \\
0
\end{array}\right| \frac{x^{3}}{\pi}+3 x^{2}\left|\begin{array}{c}
1 \\
0 \\
0
\end{array}\right|-x \pi\left|\begin{array}{l}
2 \\
1 \\
0
\end{array}\right|\right\}
\end{aligned}
$$

Straightforward computations show that the function $G(x)$ defined by (2.16) satisfies (2.30) and since $\mathrm{J}=2$, one gets

$$
\sup \left\{\left\|G^{(6)}(x)\right\| ; 0 \leq x \leq \pi\right\} \leq \frac{6.375}{2} \leq 5=M
$$


where

$$
G(x)=\frac{(x(x-\pi))^{7}}{8 ! \pi^{8}}\left|\begin{array}{c}
\frac{1}{2} \\
1 / 4 \\
\frac{1}{2}
\end{array}\right|
$$

Given the admissible error $\varepsilon=0.01$, the constant appearing in the right-hand side of (3.5) takes the value 0.06400 and since $\sum_{n \geq 1} n^{-4}=\pi^{4} / 90=1.082323234$, the integer $n_{0}$ satisfying (3.5) is the first positive integer $n_{0}$ such that

$$
1+2^{-4}+\ldots+\left(n_{0}\right)^{-4}>\pi^{4} / 90-0.06400=1.018323234
$$

In this case is $n_{0}=2$ because $1+2^{-4}=1.0625$. Taking $j=3$ one satisfies the corresponding inequality (3.7). Now in order to take the appropriate value of $q$, the positive constant $r$ must satisfy

$$
\gamma<\frac{\varepsilon}{5 \times 3^{\frac{1}{2}}(e+\exp (4))}=2.0146064 \times 10^{-5}
$$

Taking $q=3$, in accordance with (3.2), it follows that $\gamma=0.99206349 \times 10^{-5}$. Thus from the previous results the approximate solution of the problem whose error is smaller than $\varepsilon$ uniformly in the domain $I(0,1 / 4)=[0,1 / 4] \times[0, \infty[$ is given by

$$
U(x, t, 2,4)=F(x, t, 2,4)+V(x, t)-T(x)+Z(x),
$$

where

$$
\begin{gathered}
F(x, t, 2,4)=F_{44}(1, t) \sin x d_{1}+F_{44}(2, t) \sin 2 x d_{2}, \\
F_{44}(1, t)=\left[\left(N_{44}(t A / 8)\right)^{-1} N_{44}(-t A / 8)\right]^{8} \\
F_{44}(2, t)=\left[\left(N_{44}(t A / 2)^{-1} N_{44}(-t A / 2)\right]^{8}\right.
\end{gathered}
$$

$N_{44}(t A / 8)=I+0.0625 A t+1.3950892 \times 10^{-5} A^{2} t^{2}+2.3251488 \times 10^{-5} A^{3} t^{3}+1.453218 \times 10^{-7} A^{4} t^{4}$, $N_{44}(-t A / 8)=I-0.0625 A t+1.3950892 \times 10^{-5} A^{2} t^{2}-2.3251488 \times 10^{-5} A^{3} t^{3}+1.453218 \times 10^{-7} A^{4} t^{4}$, $N_{44}(t A / 2)=I+0.25 A t+2.2321427 \times 10^{-4} A^{2} t^{2}+1.48809523 \times 10^{-3} A^{3} t^{3}+3.72023808 \times 10^{-5} A^{4} t^{4}$, $N_{44}(-t A / 2)=I-0.25 A t+2.2321427 \times 10^{-4} A^{2} t^{2}-1.28809523 \times 10^{-3} A^{3} t^{3}+3.72023808 \times 10^{-5} A^{4} t^{4}$

The coefficients $d_{1}$ and $d_{2}$ defined by (2.18) may be computed in an explicit analytic way using formulas 2.634 of $[20], p .184$.

ACKNOWLEDGEMENT. This work was partially funded by the NATO grant CRG 900040 and the D.G.I.C.Y.T. grant PS90-0140.

\section{REFERENCES}

1. APOSTOL, T.M. Mathematical Analysis, Addison-Wesley, Reading, 1976.

2. CANNON, J.R. and KLEIN, R.E. On the observability and stability of the temperature distribution in a composite heat conductor, SIAM J. App1. Math. 24 (1973), 596-602. 
3. CLARKE, N.S. Heat diffusion in random laminates, J. Mech. Appl. Math. 37 (1984) 195-230.

4. COURANT, R. and HILBERT, D. Methods of Mathematical Physics Vol. II, Interscience New York, 1962.

5. CHIU, H.H. Theory of irreducible linear systems, Quartely Appl. Math. XXVII (1969), 87-104.

6. HILL, J.M. and WILLIS, J.R. Analytical aspects of heat flow in random two-component laminates, Quarterly App1. Math. Vo1. XLVI (1988), 353-364.

7. GOLUB, G.H. and VAN LOAN, C.F. Matrix Computations, John Hopkins Univ. Press, Baltimore, 1985.

8. JANKE, W., SKAGGS, W.E. and WINFREE, A.T. Chemical vortex dynamics in the Belousov Zhabotinsky reaction and in the 2-variable oregonator mode1, J. Chem. Phys. 93 (1989), 740-749.

9. JÓDAR, L. Computing accurate solutions for coupled systems of second order partial differential squations, Int. J. Computer Math. 37 (1990), 201-212.

10. LEE, A.I. and HILL, J.M. On the general linear coupled system for diffusion in media with two diffusivities, J. Math. Anal. App1. 89 (1982),530-538.

11. MOLER, C.B. and VAN LOAN, C.F. Nineteen dubious ways to compute the exponential of a matrix, SIAM Review 20 (1978), 801-836.

12. ORTEGA, J.M. and RHEINBOLDT, W.C. Iterative Solution of Nonlinear Equations in Several Variables, Academic Press, New York, 1970.

13. SEZGIN, M. Magnetohydrodynamic flow in a rectangular duct, Int. J. Numer. Meth. Fluids $\underline{7}$ (1987), 697-718.

14. TROY, W. The bifurcation of periodic solutions in the Hodgkin-Maxley equations, Quarter1y App1. Math. (1978), 73-83.

15. TROY, W. Oscillation phenomena in the Hodgkin-Maxley equations, Proc. Roy. Soc. Edinhurgh 74A (1976), 299-310.

16. ZACHMANOGLOU, E.C. and THOE, D.W. Introduction to Partial Differential Equations with Applications, Willian and Wilkins, Baltimore, 1976.

17. ZYGMUND, A. Trigonometric Series, Second ed. Vols. I and II, Cambridge Univ. Press, 1977.

18. WINFREE, A.T. When Time Breaks Down: 3-Dimensional Dynamics of Electrochemical Waves and Cardiac Arrytmias, Princeton Univ. Press, Princeton, 1987.

19. DUNFORD, $\mathrm{N}$ and SCHWARTZ, J. Linear Operators, Part I, Interscience, New York, 1957.

20. GRADSHTEYN, I.S. and RYZHIK, I.M. Table of Integrals, Series, and Products, Academic Press, New York, 1980. 


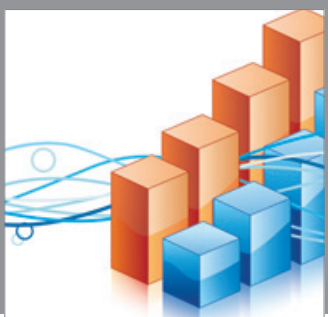

Advances in

Operations Research

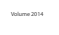

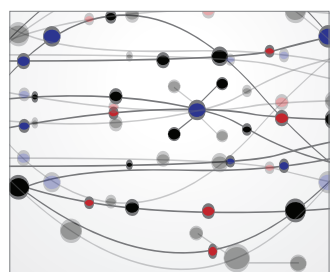

\section{The Scientific} World Journal
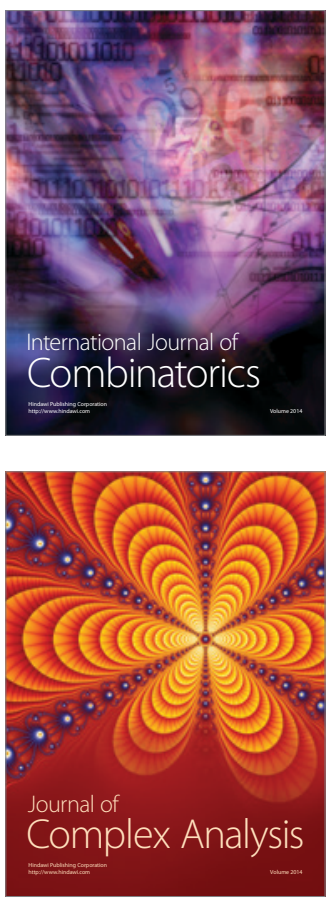

International Journal of

Mathematics and

Mathematical

Sciences
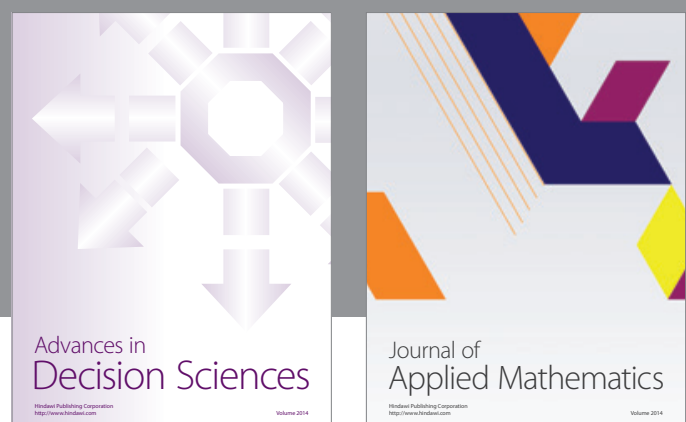

Journal of

Applied Mathematics
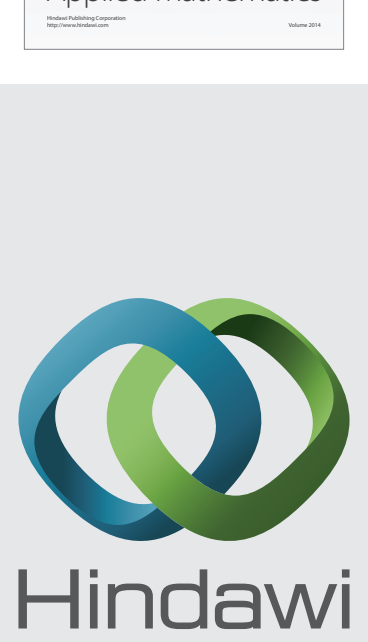

Submit your manuscripts at http://www.hindawi.com
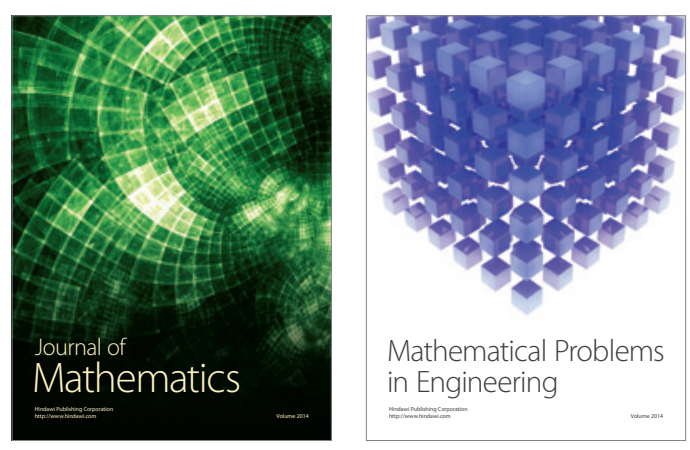

Mathematical Problems in Engineering
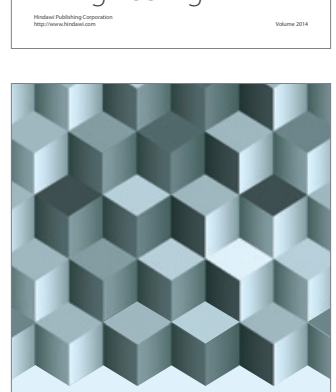

Journal of

Function Spaces
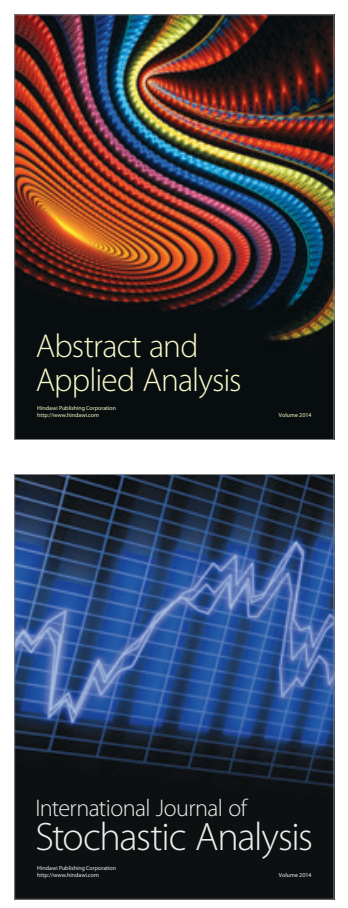

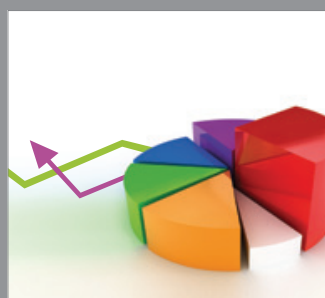

ournal of

Probability and Statistics

Promensencen
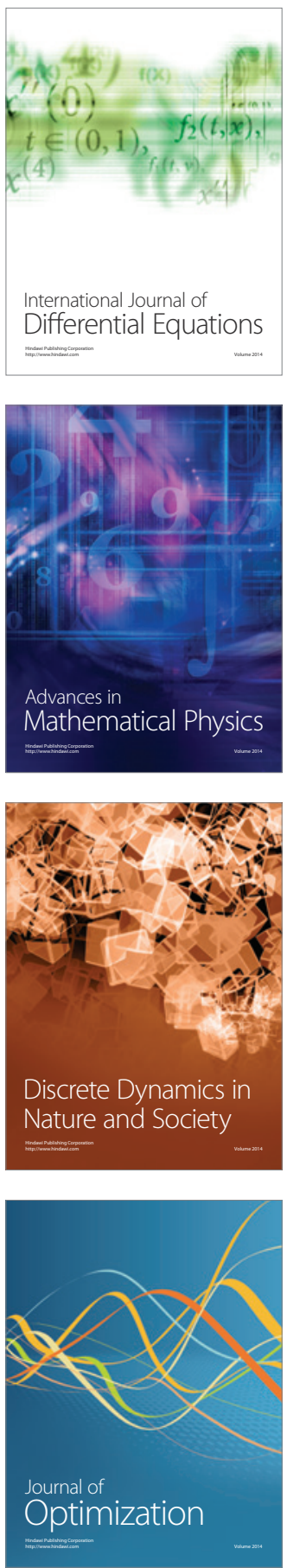